\title{
Phonetic analysis during treatment with rapid maxillary expander
}

\author{
E. Biondi ${ }^{1, \dagger}$ \\ A. Bandini ${ }^{2,3, \dagger}$ \\ L. Lombardo ${ }^{1}$ \\ S. Orlandi ${ }^{2}$ \\ G. Siciliani ${ }^{1}$ \\ C. Manfredi ${ }^{2}$
}

\author{
${ }^{1}$ Department of Orthodontics, University of \\ Ferrara, Ferrara, Italy \\ ${ }^{2}$ Department of Information Engineering, \\ University of Firenze, Firenze, Italy \\ ${ }^{3}$ Department of Electrical, Electronic and \\ Information Engineering (DEI) "Guglielmo \\ Marconi", University of Bologna, Bologna, Italy

\section{Correspondence} \\ E. Biondi, Department of Orthodontics, \\ University of Ferrara, Ferrara, Italy. \\ Email: eleonora.biondi@hotmail.it
}

\section{Structured Abstract}

Objectives: To investigate possible changes and/or device-related impairments in phonetic habits produced by rapid maxillary expansion (RME).

Materials and Methods: Thirty-five patients scheduled for RME were divided into two groups: Group A (banded two-arm Hyrax) and Group B (banded four-arm Hyrax). Speech samples were collected at six time points, before, during and after RME removal. Acoustical analysis was performed using PRAAT and BioVoice analysis tools. Ten volunteers completed a questionnaire on the acceptability of patient's speech. Maxillary dimensions and palatal volume were measured on dental casts before and after expansion using a digital gauge.

Results: Voice analysis showed an increase in the peak frequency of fricative consonants (/s/,/J/) after expansion, whereas there was no change of formant frequencies of palatal consonants $(/ \mathrm{n} /, / / /)$. Vowel $/ \mathrm{i} /$ displayed a lowering of the first formant frequency, and an increase in the second and third formant frequencies. After bonding, Group B showed both a greater reduction in the peak frequency of fricatives and a greater increase in the formant frequencies of palatal consonants than Group A.

Conclusion: Rapid maxillary expansion causes a slight phonetic change in the acoustical parameters of both consonants and vowels. The two-arm Hyrax caused less speech impairment than the four-arm Hyrax during the treatment.

KEYWORDS

maxillary expansion, palate, phonetics, speech acoustic

\section{1 | INTRODUCTION}

Rapid maxillary expansion (RME) is an effective treatment widely used in orthodontics; it does not significantly alter the height of the palatal vault, ${ }^{1,2}$ but it can increase palatal volume up to $21 \% .^{3}$ These significant changes in palatal morphology may affect speech by modifying the articulation sites of the tongue on the palate and changing the oral resonance mechanism by enlarging the oral cavity. A correct speech pronunciation is related to palate size, ${ }^{4}$ and maxillary surgical expansion causes vowel sound modification. ${ }^{5}$ The introduction of fixed

${ }^{\dagger}$ These authors contributed equally. elements in the oral cavity, such as an artificial palate or orthodontic appliances, alters consonant and vowel articulation.,

Although several orthodontics-related phonetics studies have been published in recent decades, ${ }^{8-10}$ only two of them focused on the alterations caused by the RME. ${ }^{11,12}$ De Felippe et al., ${ }^{11}$ investigated the impact of RME on speech relying on patients' perception and selfassessment questionnaires, demonstrating that patients perceive that RME affects their speech. Stevens et al., ${ }^{12}$ performed an acoustical analysis assessing RME-induced speech perturbations over time. The authors demonstrated that the speech returned to baseline level at RME removal. Speech acceptability ratings after treatment were better than before expansion. The latter findings come from questionnaire surveys only. Therefore, we propose a study based on objective 
acoustical parameters to assess phonetical changes induced by RME. Specifically, we compare pre- and post-expansion parameters related to the oral cavity. Moreover, we investigate possible differences between two-arm ${ }^{13-15}$ and four-arm Hyrax RMEs (Figure 1) to assess whether the bulkier device interferes with speech to a greater degree.

\section{2 | MATERIALS AND METHODS}

Thirty-five patients scheduled for RME who had no previous speech therapy (21 females, 14 males, age 7-14 years, mean 9.3 \pm 2.3 years) were recruited. Each subject underwent panoramic radiography, lateral cephalogram and cephalometric analysis. Patients were divided into two groups, depending on the device: $\mathrm{n}=26$ in Group A (banded 2-arm Hyrax RME Philosophy ${ }^{\circledR}$, Lancer Italia S.r.l.,Trezzano sul Naviglio (Milano), Italy) and $n=9$ in Group B (banded 4-arm Hyrax RME Philosophy ${ }^{\circledR}$, Lancer Italia S.r.l., Trezzano sul Naviglio (Milano), Italy) (Figure 1). RMEs were activated using $12.5-\mathrm{mm}$ or $14.5-\mathrm{mm}$ screws, depending on the best fit to the subject's palate. The devices were activated using the same protocol: a single turn of screw $(0.20 \mathrm{~mm})$ per day, until the palatal cusp of the upper first molar came into contact with the buccal cusp of the lower first molar. All subjects were native speakers in Italian.

Dental casts taken before and after expansion were scanned using a RevengOrthodontic professional 3D scanner (Nemotec, Sarzana (Spezia), Italy). Four linear maxillary distances were measured on the scanned models through the software Rhinoceros ${ }^{\circledR}$ : intercanine distance, intermolar distance, cuspid and molar heights. ${ }^{16,17}$ The palatal volume was measured as the area contained within a horizontal plane passing through the lowest gingival point of one central incisor and the first permanent molars, and a vertical plane tangent to the distal surfaces of the first molars, perpendicular to the horizontal plane. ${ }^{3}$

Speech samples were collected with Audacity software, Boston, MA, USA (version 2.0.3) using a high-quality microphone (Go Mic, Samson, Hauppauge, NY, USA) connected to a laptop. Signals were sampled at $44.1 \mathrm{kHz}$ and stored in 16-bit wav files. All samples were recorded in a noise-free room with the microphone placed $5 \mathrm{~cm}$ below the patient's chin, orientated $45^{\circ}$ forwards and downwards. Fortythree Italian sentences were chosen by a phonetics specialist. The speech task consisted in three repetitions of each sentence, and ten repetitions of the vowel /i/, chosen because it requires a high position of the tongue, making it the most affected vowel by changes in palate morphology. According to Stevens et al., 2011, ${ }^{12}$ recordings were made at six time points: before (T0), 15 minutes after (T1), 1 month after (T2) and 3 months after the RME fitting (T3), 6 months after fitting (T4) and 2 months after the RME removal (T5).

From the corpus, a sample sentence was selected for perceptive analysis. A group of 10 listeners, with no prior knowledge of phonetics or speech therapy and unaware of the aim of this study, were trained to judge the acceptability of patients' speech according to a Likert scale. ${ }^{18}$ Listeners gave to each sample a score ranging from 1 to 5 (1-proper pronunciation, 5-severely altered pronunciation). Pre-treatment (T0) scores were used to classify subjects as either "normal speakers" (score 1-1.9) or "people with pre-existing speech difficulties" (score $>2$ ).

Among the sentences, those containing the consonants involving the greatest contact of the tongue on the hard palate were chosen: fricatives /s/, / $/$ / and palatal /n/, / $/$.

Two kinds of acoustical analysis were performed:

1. The analysis of phonetic changes during and after RME therapy. We analysed fricatives $(/ \mathrm{s} /, / \mathrm{J} /)$ and palatal $(/ \mathrm{n} /, / / /)$ consonants extracted from four sentences and uttered by 10 patients fitted with the four-arm appliance, chosen randomly within the group. Three sentence repetitions were considered for each time step (720 overall samples). We also analysed repetitions of the vowel /i/ at each recording step (600 overall samples).

2. Comparison between the two kinds of RME. The analysis of fricatives $(/ \mathrm{s} /, / \mathrm{S} /)$ and palatal $(/ \mathrm{n} /, / \mathrm{N} /)$ consonants extracted from the same four sentences was performed during the three repetitions, uttered by 13 patients (six from Group A, seven from Group B, chosen randomly) at $\mathrm{T} 1$, just after bonding, when the speech impairment is greatest.

Consonants and vowels were manually extracted from the corpus. After amplitude normalization, the power spectral density (PSD) was estimated on 128-point windows with the Welch method. The following parameters were computed from the PSD:

- Power percentage (ratio of the spectral power within the frequency bands of interest and the overall spectral power) in: low $(2.5-8 \mathrm{kHz}-$ $\left.\mathrm{P}_{\text {If }} \%\right)$ and high frequency band $\left(5-15 \mathrm{kHz}-\mathrm{P}_{\mathrm{hf}} \%\right)$;

- Peak frequency $[\mathrm{Hz}]$ : maximum value of the PSD within the frequency ranges of interest;

- PSD spectral moments (variance, skewness, kurtosis) of fricatives.
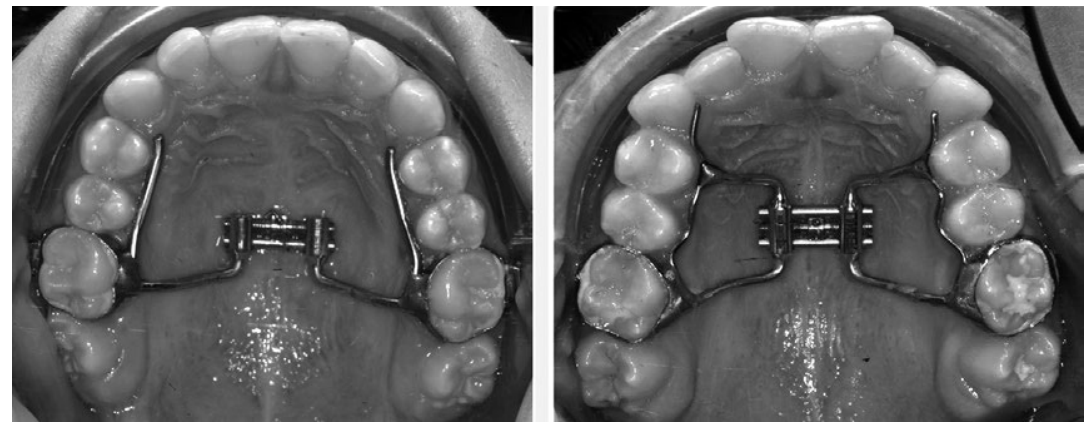

FIGURE 1 Two-arm RME on the left: four-arm RME on the right 
These parameters were calculated according to REF., ${ }^{19,20}$ where authors observed that $/ \int /$ and $/ \mathrm{s} /$ have the spectral peak around $3 \mathrm{kHz}$ and 4-5 kHz, respectively. However, those studies were performed on adult speakers. As higher peak frequencies are expected in children, we considered larger frequency bands (/ $/: 2.5-8 \mathrm{kHz}, / \mathrm{s} /: 5-15 \mathrm{kHz})$. To exclude contributions due to vowels adjacent to the considered consonant (predominant at frequencies $<2.5 \mathrm{kHz}$ ), peak values were chosen as an approximation of the first spectral moment. A customized software written in Matlab language (ver. 2012a) (The Mathworks Inc., Natick, MA, United States), was developed for the analysis of fricatives consonants.

Concerning vowels and palatal consonants, the first three formant frequencies (F1-F3) were estimated through BioVoice ${ }^{21}$ (a software developed for adult voice ${ }^{22}$ and newborn cry analysis ${ }^{23,24}$ ) and PRAAT. ${ }^{25}$ BioVoice allows the sequential analysis of several audio signals at once without any manual setting. Formants F1-F3 are obtained by peak selection from a parametric PSD (AR models), whose variable order is estimated on time windows of variable length. PRAAT implements a method based on autocorrelation, applied to a time window of fixed size, and linear predictive coding. It requires the manual setting of some parameters. Therefore, we tested and set the best parameters to maximize the reliability of results.

\section{1 | Statistical analysis}

The average gain in linear measurements and volume produced RME was calculated on digital models. Digital model measurements and acoustical parameters pertaining to the two kinds of RME (two and four arms) were compared by analysis of variance (ANOVA) and $t$ test. $\mathrm{K}$-means cluster analysis was used to divide patients into three groups based on their linear and volumetric palate dimensions (small, medium and large), and average acceptability ratings were calculated from the questionnaire. Statistical analysis of phonetic results was performed using Matlab R2012a, The Mathworks Inc., Natick, MA, United States and Microsoft Excel 2010, Microsoft, Redmond, WA, USA.

\section{3 | RESULTS}

3D measurement of digital models is presented in Table 1. Patients were clustered into three groups based on pre-expansion palate size: small (15 subjects), medium (15 subjects) and large (five subjects). Group B (four-arm RME) showed a greater increase in intercanine distance, intermolar distance and volume than Group A (two-arm RME), but differences were not statistically significant.

The questionnaire scores showed a perceived worsening of speech after RME placement, followed by a gradual improvement at T2 and T3. At T4, respondents noted again a speech impairment, with a return to pre-treatment level at T5. Group B displayed a greater speech impairment than Group A immediately after bonding (T1). Eight children were judged with "pre-existing speech difficulties."

Acoustical parameters used to study phonetic changes during and after RME therapy are reported in Table 2. Acoustical parameters of fricatives consonants are shown in Figure 2, while formant frequencies extracted from palatal consonants and vowels are reported in Figure 3. Peak frequency of fricatives decreased from T0 to T1, at bonding, increased at T2 and returned to baseline at T3. At T5, it reached values greater than those registered at TO (Figure 2A). Skewness and kurtosis showed similar trends (especially for $/ \mathrm{s} /$ ) with values close to zero at T5 and lower than those observed at T0 (Figure 2C).

Palatal consonants and vowel /i/ were analysed with PRAAT and BioVoice. Both tools showed that F1 and F3 of palatal consonants remained stable (albeit fluctuations) over time (Figure 3). PRAAT showed that F2 of palatal consonants increased from T0 to T2, returning to the T0 values at T5. The nasal palatal /n/ had higher F2 values than the lateral $/ N /$ throughout the observation period. Furthermore, F2 of / $\mathrm{n} /$ decreased progressively from T2 to T5, while F2 / $/$ / started to decrease after T3 (Figure 2, Table 2). Similar trends of formant frequencies were found with both tools. Vowel /i/ underwent a centralization (F1 increases, F2 decreases) effect after bonding (T1). At T5, F1 was lower, while F2 and F3 were higher than baseline values (Table 2, Figure 3).

Results on the comparison between the two kinds of RME at T1 are reported in Table 3. Questionnaire results were confirmed by the acoustical analysis: Group B showed lower peak frequency for fricatives and lower variance for /s/. Moreover, Group B showed a higher F1 of palatal consonants than Group A.

\section{4 | DISCUSSION}

Our findings confirm other studies ${ }^{5,7,9}$ showing that placement of an orthodontic device causes an immediate reduction in fricative peak frequency. Both fricatives displayed this behaviour at RME placement, and then, the peak frequency gradually increased during therapy. After device removal, the peak frequency dropped, presumably due to temporary tongue disorientation, and then increased again to a value higher than pre-treatment one.

The spectral variance of fricatives increased from $\mathrm{TO}$ to $\mathrm{T} 5$, while the skewness decreasing (close to zero at T5) reflects an increase in the spectral peak indicating a shift towards higher peak frequencies. Kurtosis also decreased from T0 to T5 reflecting an increase in standard deviation and therefore a flatter spectrum.

At the end of maxillary expansion, the PSDs of both fricatives were more homogeneous and skewed, indicating that the peak was more stable but at a higher frequency. This is somewhat at odds with our finding that the maxillary expansion caused an increase in palatal volume, which should lead to a reduction in frequency. However, Iwasaki et al., ${ }^{26}$ demonstrated that the tongue position changes after RME, moving higher in the palatal vault, thus creating 


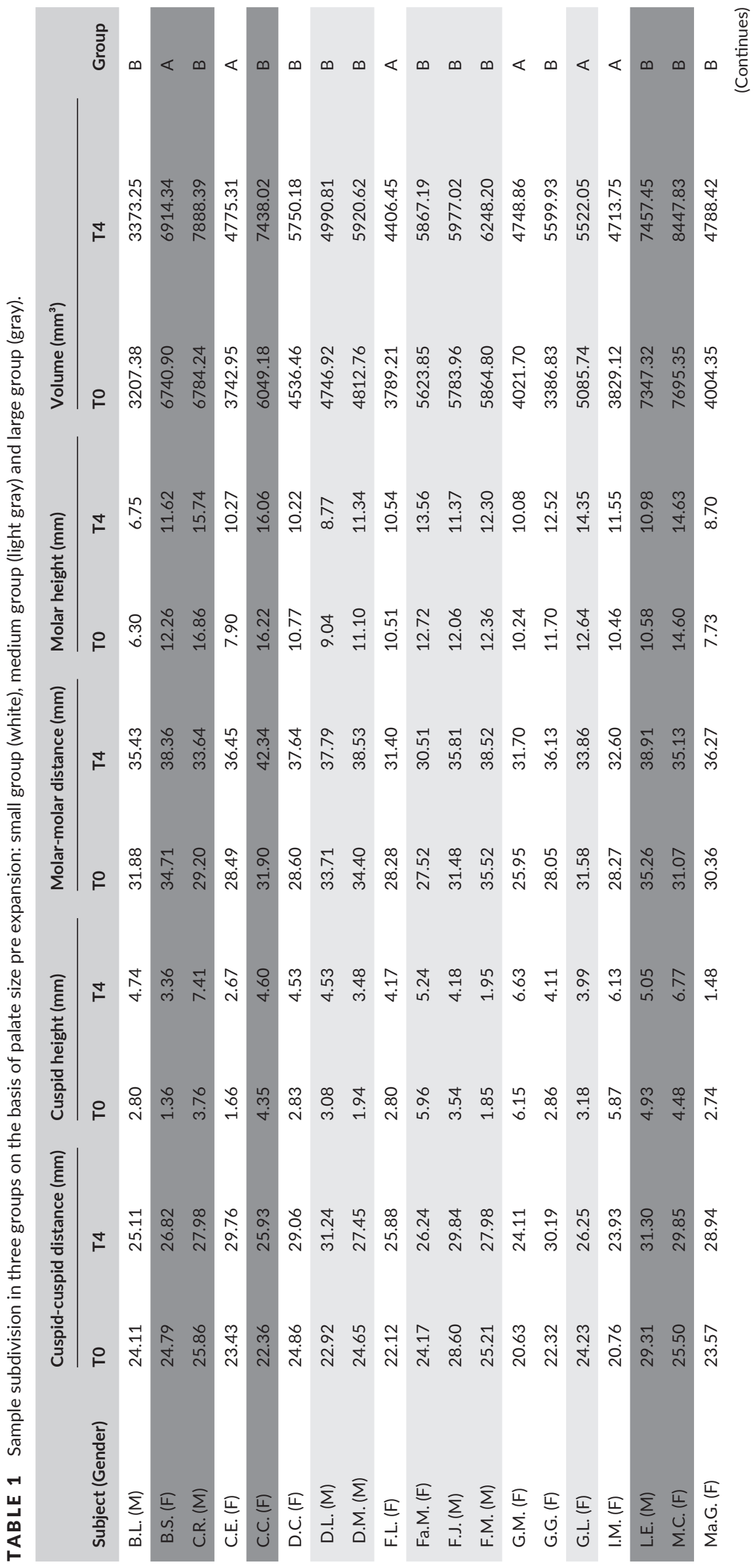



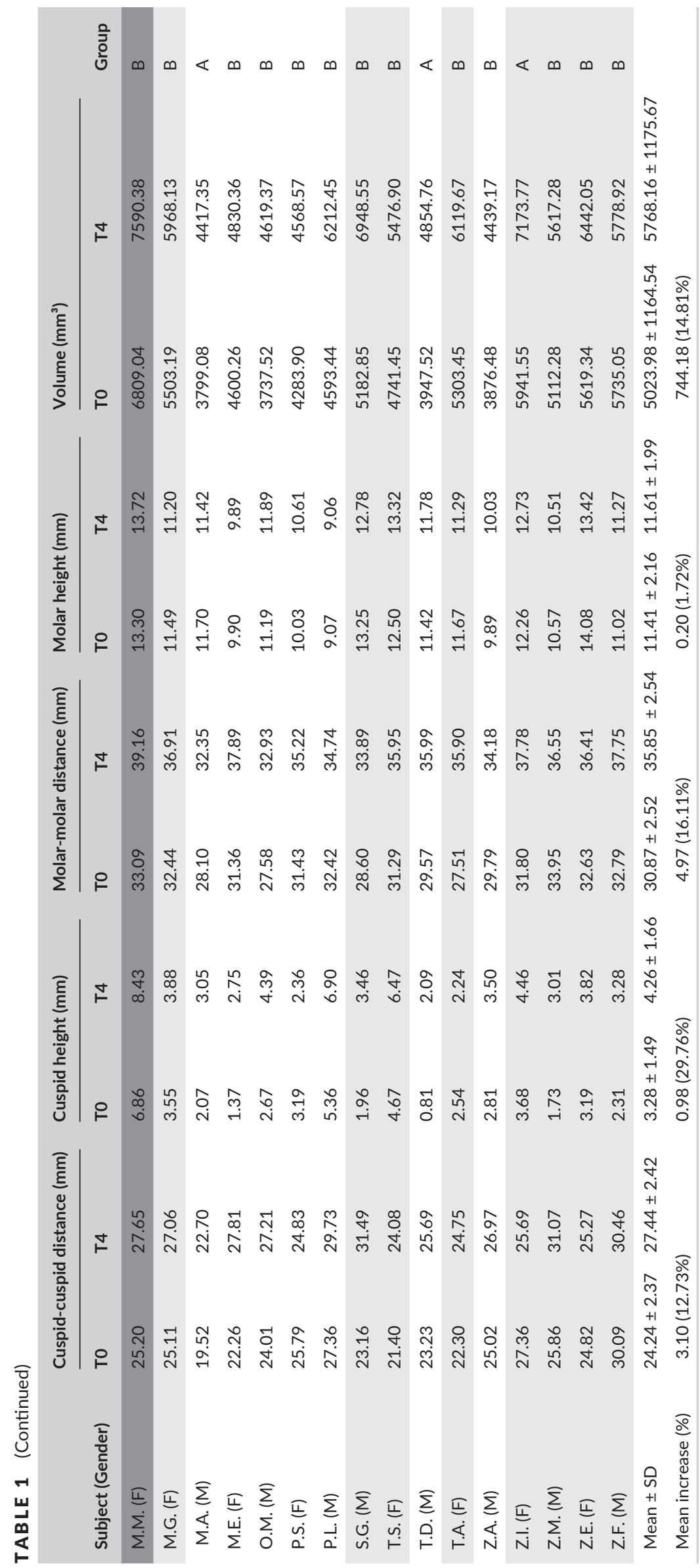


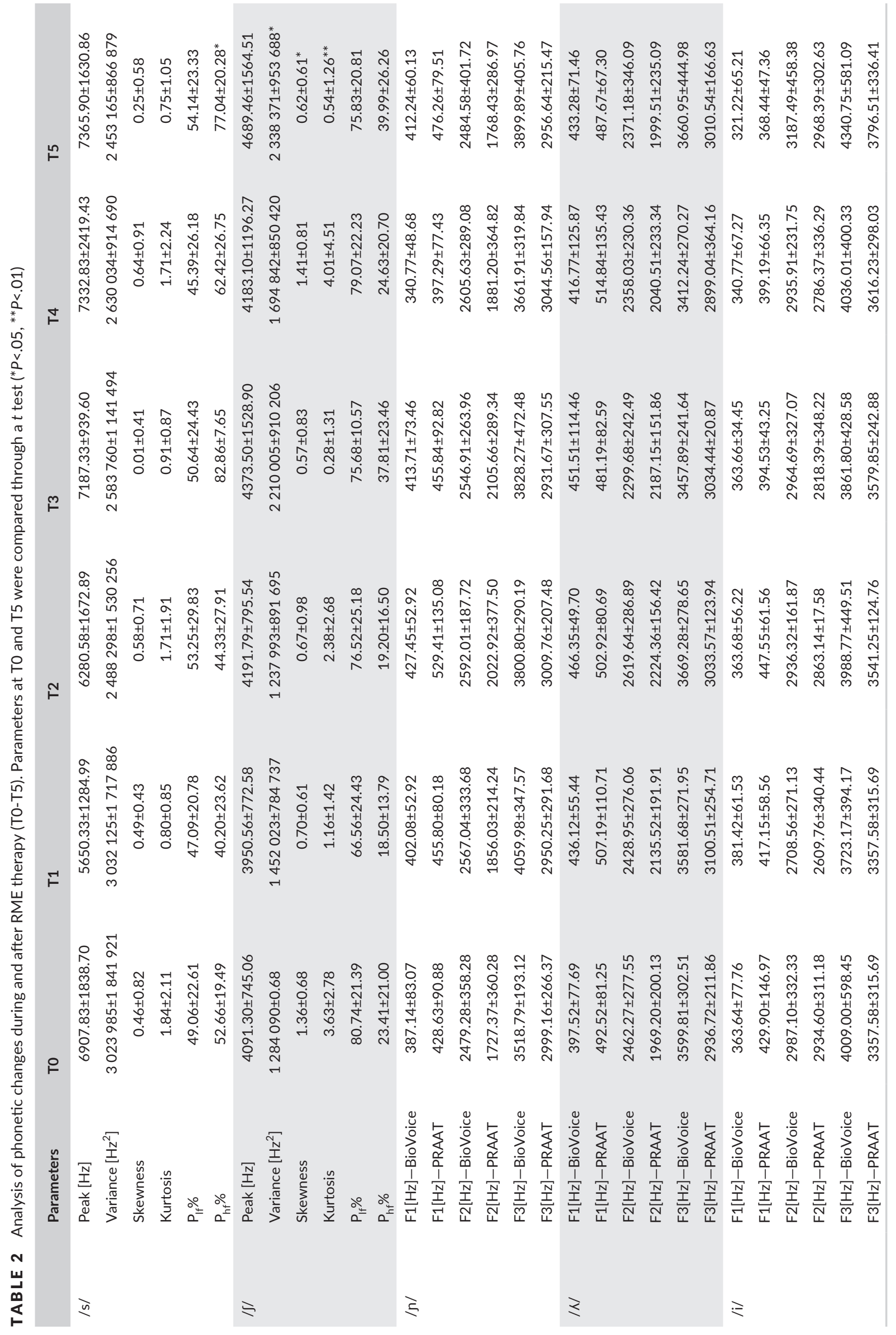



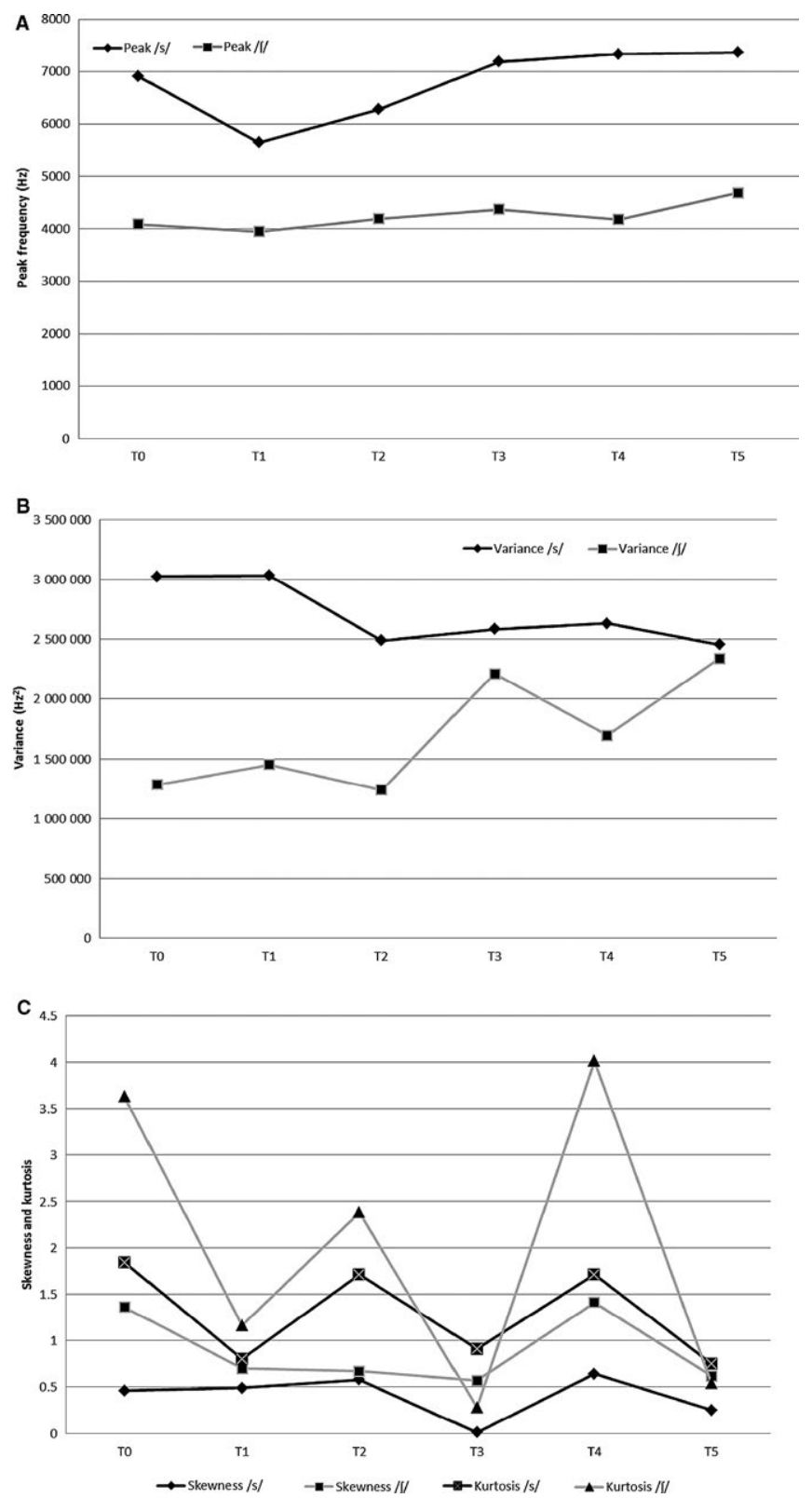

FIGURE 2 Acoustical parameters of fricatives: (A) peak; (B) variance; (C) skewness, kurtosis

a smaller resonance cavity between the top of the tongue and the palatal vault.

In the literature, there are no investigations regarding palatal consonants. Nevertheless, two studies ${ }^{27,28}$ demonstrated that after surgical augmentation of the upper airways, formant frequencies of both vowels and nasal consonants are reduced. Likewise, Ungor et al., ${ }^{29}$ reported that after surgical reduction in the paranasal sinus, F1 of nasal vowels decreased, whereas F2 and F3 increased; in fact, if a constriction in the palatal region occurs, F2 and F3 will have higher values, whereas a higher value of F1 requires a larger oral cavity. ${ }^{30}$

In our analysis of palatal consonants, F1 remained stable from TO to T5 in both, while F2 and F3 showed slight changes, corresponding to the perturbation effects of the RME. However, at RME removal, no
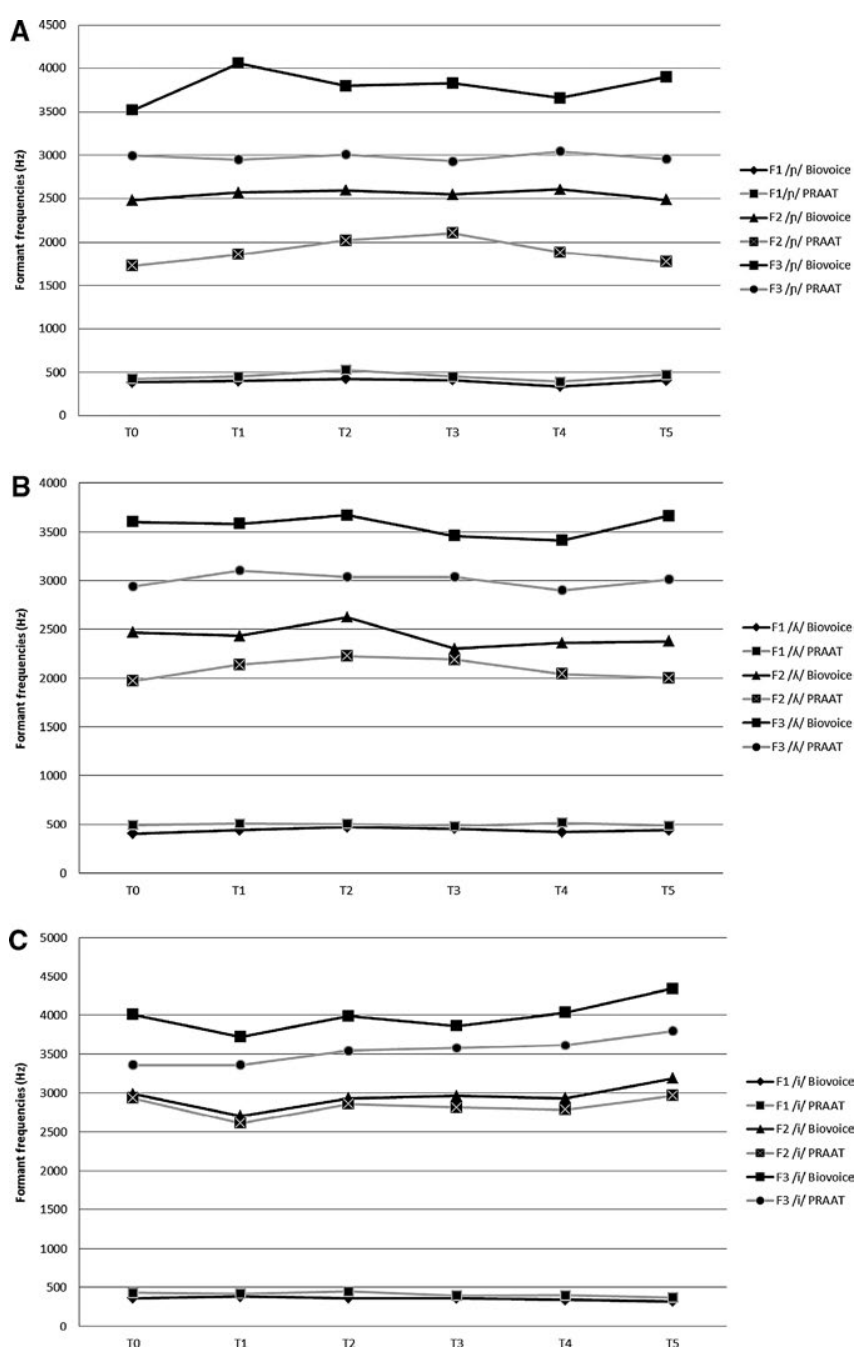

FIGURE 3 Formant frequencies of: (A) nasal palatal; (B) lateral palatal; (C) vowel /i/

significant differences were found between T0 and T5. In contrast to results obtained for fricatives, for palatal consonants it was possible to note only the perturbation caused by the device.

In the vowel sound /i/, the perturbation introduced by the RME caused a centralization of $\mathrm{F} 1$ and $\mathrm{F} 2$ frequencies at $\mathrm{T} 1$; the resonance changed when the device was inserted into the oral cavity. This finding was in line with the centralization found in. ${ }^{5}$

Group B (four-arm RME) reported lower a peak for fricatives and higher formant values of palatal consonants, both signs of speech worsening found in T0-T1 comparison.

\section{5 | CONCLUSION}

This study shows that in growing children, RME therapy causes modification of both fricatives and the vowel sound /i/, while palatal consonants do not change significantly. The modifications correspond to a reduction in the volume of resonance cavities after RME, confirming that tongue moves higher in the oral cavity, closer to the palate. The 


\begin{tabular}{|c|c|c|c|c|}
\hline & Parameters & $\begin{array}{l}\text { Group A (two-arm } \\
\text { RME) }\end{array}$ & $\begin{array}{l}\text { Group B (four-arm } \\
\text { RME) }\end{array}$ & $t$ test \\
\hline \multirow[t]{6}{*}{$/ \mathrm{s} /$} & Peak $[\mathrm{Hz}]$ & $5603.11 \pm 1209.38$ & $4801.43 \pm 922.44$ & $P=.03$ \\
\hline & Variance $\left[\mathrm{Hz}^{2}\right]$ & $3294789 \pm 2097408$ & $1820162 \pm 825612$ & $P=.01$ \\
\hline & Skewness & $0.96 \pm 0.82$ & $1.35 \pm 0.58$ & $P=.11$ \\
\hline & Kurtosis & $2.03 \pm 3.90$ & $2.85 \pm 2.59$ & $P=.46$ \\
\hline & $\mathrm{P}_{\text {If }} \%$ & $17.18 \pm 14.91$ & $26.59 \pm 20.20$ & $P=.10$ \\
\hline & $\mathrm{P}_{\mathrm{hf}} \%$ & $10.20 \pm 10.21$ & $9.33 \pm 11.23$ & $P=.80$ \\
\hline \multirow[t]{6}{*}{$/ \mathrm{s} /$} & Peak $[\mathrm{Hz}]$ & $4261.26 \pm 1082.29$ & $3445.18 \pm 756.15$ & $P=.01$ \\
\hline & Variance $\left[\mathrm{Hz}^{2}\right]$ & $1393855 \pm 854590$ & $1205211 \pm 731315$ & $P=.47$ \\
\hline & Skewness & $1.19 \pm 0.84$ & $1.14 \pm 0.56$ & $P=.81$ \\
\hline & Kurtosis & $2.65 \pm 2.23$ & $2.23 \pm 2.86$ & $P=.61$ \\
\hline & $\mathrm{P}_{\mathrm{lf}} \%$ & $46.16 \pm 25.04$ & $60.63 \pm 21.13$ & $P=.06$ \\
\hline & $\mathrm{P}_{\mathrm{hf}} \%$ & $13.24 \pm 12.97$ & $9.10 \pm 9.13$ & $P=.27$ \\
\hline \multirow[t]{6}{*}{$/ \mathrm{n} /$} & F1 $[\mathrm{Hz}]$-BioVoice & $309.88 \pm 97.50$ & $418.78 \pm 164.88$ & $P=.02$ \\
\hline & F1 $[\mathrm{Hz}]-P R A A T$ & $433.77 \pm 177.28$ & $511.16 \pm 108.65$ & $P=.12$ \\
\hline & $\mathrm{F} 2[\mathrm{~Hz}]-$ BioVoice & $1913.98 \pm 764.09$ & $2286.97 \pm 341.32$ & $P=.08$ \\
\hline & $\mathrm{F} 2[\mathrm{~Hz}]-\mathrm{PRAAT}$ & $1626.81 \pm 544.26$ & $1889.97 \pm 290.79$ & $P=.08$ \\
\hline & F3 $[\mathrm{Hz}]-$ BioVoice & $3057.81 \pm 927.74$ & $3407.81 \pm 466.17$ & $P=.17$ \\
\hline & F3[Hz]-PRAAT & $2486.52 \pm 724.27$ & $2867.61 \pm 221.97$ & $P=.04$ \\
\hline \multirow[t]{6}{*}{ IN/ } & F1 $[\mathrm{Hz}]-$ BioVoice & $363.05 \pm 109.15$ & $443.21 \pm 74.27$ & $P=.03$ \\
\hline & F1[Hz]-PRAAT & $514.68 \pm 108.29$ & $516.20 \pm 65.70$ & $P=.96$ \\
\hline & F2 $[\mathrm{Hz}]-$ BioVoice & $2629.70 \pm 681.30$ & $2304.27 \pm 355.19$ & $P=.12$ \\
\hline & $\mathrm{F} 2[\mathrm{~Hz}]-\mathrm{PRAAT}$ & $1954.03 \pm 519.93$ & $1961.21 \pm 235.42$ & $P=.96$ \\
\hline & F3 $[\mathrm{Hz}]-$ BioVoice & $3734.70 \pm 583.65$ & $3403.83 \pm 437.93$ & $P=.09$ \\
\hline & F3[Hz]-PRAAT & $2920.63 \pm 591.99$ & $2947.11 \pm 215.23$ & $P=.86$ \\
\hline
\end{tabular}

TABLE 3 Comparison between the two kinds of RME (two-arm and four-arm RME) immediately after bonding (T1) speech impairment was greater with a four-arm RME, although this difference is meaningful only during the first 3 months of application. Therefore, when a massive expansion is not strictly necessary, clinicians can choose a two-arm RME which gives less phonetic impairments.

\section{CONFLICT OF INTEREST}

The authors have stated explicitly that there are no conflict of interests in connection with this article.

\section{REFERENCES}

1. Lagravere MO, Major PW, Flores-Mir C. Long-term skeletal changes with rapid maxillary expansion: a systematic review. Angle Orthod. 2005;75:1046-1052.

2. Lagravere MO, Major PW, Flores-Mir C. Long-term dental arch changes after rapid maxillary expansion treatment: a systematic review. Angle Orthod. 2005;75:155-161.

3. Gracco A, Malaguti A, Lombardo L, Mazzoli A, Raffaeli R. Palatal volume following rapid maxillary expansion in mixed dentition. Angle Orthod. 2010;80:153-159.

4. Laine T. Articulatory disorders in speech as related to size of the alveolar arches. Eur J Orthod. 1986;8:192-197.

5. Sari E, Kilic MA. The effects of surgical rapid maxillary expansion (SRME) on vowel formants. Clin Linguist Phon. 2009;23:393-403.
6. McFarland DH, Baum SR, Chabot C. Speech compensation to structural modification of the oral cavity. J Acoust Soc Am. 1996;100:1093-1104.

7. Baum SR, McFarland DH. The development of speech adaptation to an artificial palate. J Acoust Soc Am. 1997;102:2353-2359.

8. Hohoff A, Seifert E, Fillion D, Stamm T, Heinecke A, Ehmer U. Speech performance in lingual orthodontic patients measured by sonography and auditive analysis. Am J Orthod Dentofacial Orthop. 2003;123:146-152.

9. Kulak Kayikci ME, Akan S, Ciger S, Ozkan S. Effects of Hawley retainers on consonants and formant frequencies of vowels. Angle Orthod. 2012;82:14-21.

10. Khattab TZ, Farah H, Al-Sabbagh R, Hajeer MY, Haj-Hamede Y. Speech performance and oral impairments with lingual and labial orthodontic appliances in the first stage of fixed treatment. A randomized controlled trial. Angle Orthod. 2013;83:519-526.

11. De Felippe NL, Da Silveira AC, Viana G, Smith B. Influence of palatal expanders on oral comfort, speech, and mastication. Am J Orthod Dentofacial Orthop. 2010;137:48-53.

12. Stevens K, Bressman T, Gong S, Tompson BD. Impact of a rapid palatal expander on speech articulation. Am J Orthod Dentofacial Orthop. 2011;140:67-75.

13. Schneidman E, Wilson S, Eriks R. Two point rapid palatal expansion: an alternative approach to traditional treatment. Ped Dent. 1990;12:92-97.

14. Lamparski DG, Rinchuse DJ, Close JM, Sciote JJ. Comparison of skeletal and dental changes between 2-point and 4-point rapid palatal expanders. Am J Orthod Dentofac Orthop. 2003;123:321-328. 
15. Bratu DC, Bratu EA, Popa G, Luca M, Balan R, Ogodescu A. Skeletal and dentoalveolar changes in maxillary bone morphology using twoarm maxillary expander. Rom J Morphol Embryol. 2012;53:35-40.

16. Phatouros A, Goonewardene MS. Morphologic changes of the palate after rapid maxillary expansion: a 3-dimensional computed tomography evaluation. Am J Orthod Dentofacial Orthop. 2008;134:117-124.

17. Muchitsch AP, Winsauer $H$, Wendl B, et al. Remodelling of the palatal dome following rapid maxillary expansion (RME): laser scan quantification during a low growth period. Orthod Craniofac Res. 2012;15:30-38.

18. Likert R. Technique for the measure of attitudes. Arch Psychol. 1932;22:1-55.

19. Forrest K, Weismer G, Milenkovic P, Dougall RN. Statistical analysis of word-initial voiceless ostruents: preliminary data. J Acoust Soc Am. 1988;84:115-123.

20. Jongman A, Wayland R, Wong S. Acoustic characteristics of English fricatives. J Acoust Soc Am. 2000;108:1252-1263.

21. Manfredi C, Bocchi L, Cantarella G. A multipurpose user-friendly tool for voice analysis: application to pathological adult voices. Biomed Signal Process Control. 2009;4:212-220.

22. Bandini A, Giovannelli F, Orlandi S, et al. Automatic identification of dysprosody in idiopathic Parkinson's disease. Biomed Signal Process Control. 2015;17:47-54.

23. Manfredi C, Bocchi L, Orlandi S, Spaccaterra L, Donzelli GP. Highresolution cry analysis in preterm newborn infants. Med Eng Phys. 2009;31:528-532.

24. Orlandi S, Reyes Garcia CA, Bandini A, Donzelli GP, Manfredi C. Application of pattern recognition techniques to the classification of full-term and preterm infant cry. J Voice. 2016;30:656-663.
25. Boersma P. Acoustic Analysis. In: Podesva RJ, Sharma D, eds. Research Methods in Linguistics. Cambridge, UK: Cambridge University Press; 2014:375-396.

26. Iwasaki T, Saitoh I, Takemoto Y, et al. Tongue posture improvement and pharyngeal airway enlargement as secondary effects of rapid maxillary expansion: a cone-beam computed tomography study. Am J Orthod Dentofacial Orthop. 2013;143:235-245.

27. Berhman A, Shikowitz MJ, Dailey S. The effects of upper airway surgery on voice. Otolaryngol Head Neck Surg. 2002;127:36-42.

28. Bertino G, Matti E, Migliazzi S, Pagella F, Tinelli C, Benazzo M. Acoustic changes in voice after surgery for snoring: preliminary results. Acta Otorinolaryngol Ital. 2006;26:110-114.

29. Ungor C, Saridogan C, Yilmaz M, Tosun E, Senel FC, Icten O. An acoustical analysis of the effects of maxillary sinus augmentation on voice quality. Oral Surg Oral Med Oral Pathol Oral Radiol. 2013;115: 175-184.

30. Harrington J. Acoustic Phonetics. In: Hardcastle WJ, Laver J, Gibbon FE, eds. The Handbook of Phonetics Sciences. Chichester, West Sussex, UK: Wiley-Blackwell; 2010.

How to cite this article: Biondi E, Bandini A, Lombardo L, Orlandi S, Siciliani G, and Manfredi C. Phonetic analysis during treatment with rapid maxillary expander. Orthod Craniofac Res. 2017;20:21-29. doi:10.1111/ocr.12136. 